\title{
Studies of Ploidy Assessment in Some Synthetic Hybrids of Banana (Musa spp.)
}

\author{
Sukhen Chandra Das ${ }^{1 *}$, T.N. Balamohan ${ }^{2}$, K. Poornima ${ }^{3}$ and I. Van den Bergh ${ }^{4}$ \\ ${ }^{1}$ Department of Horticulture, College of Agriculture, Tripura, India \\ ${ }^{2}$ Department of Fruit Crop, HC and RI, TNAU, Tamil Nadu, India \\ ${ }^{3}$ Department of Nematology, HC and RI, TNAU, Tamil Nadu, India \\ ${ }^{4}$ International Network for the Improvement of Banana and Plantain-Asia and the Pacific, c/o \\ IRRI Khush Hall, Los Banos, Laguna 4031, Philippines \\ *Corresponding author
}

A B S T R A C T

\begin{tabular}{|c|}
\hline Keywords \\
\hline $\begin{array}{l}\text { Banana, Hybrids, } \\
\text { Ploidy assessment }\end{array}$ \\
\hline Article Info \\
\hline $\begin{array}{l}\text { Accepted: } \\
\text { 17 July } 2018 \\
\text { Available Online: } \\
\text { 10 August } 2018\end{array}$ \\
\hline
\end{tabular}

Banana (Musa spp.) constitute a hybrid-polyploid complex and are classified according to different genome compositions such as AA, BB, AB, AAA, AAB, ABB, AAAA, ABBB, $\mathrm{AAAB}, \mathrm{AABB}$ and $\mathrm{AAABB}$. Knowledge of ploidy and exact genome compositions of the parental material is essential for Musa breeding. Flow cytometric analysis of nuclear DNA content was used to estimate ploidy levels. Twenty four Banana hybrids under phase-I and nineteen hybrids under phase-II evaluated and was done by flow cytometry analysis which enables rapid and precise measurements on whole cells, isolated nuclei or chromosomes in a monodisperse suspension. Studies found under phase-I that six hybrids diploids (AA and $\mathrm{AB}$ ), five hybrids triploids (AAA and $\mathrm{AAB}$ ), ten hybrids tetraploids (AABB) and three hybrids pentaploids (AAABB) were recorded and under phase-II found that one hybrid diploids $(\mathrm{AB})$, three triploids $(\mathrm{AAB})$ and rest of the hybrids tetraploids $(\mathrm{AABB})$ were recorded.

\section{Introduction}

Bananas are among the largest herbs in the world. They are perennials with tall aerial shoots that arise from swollen, fleshy corms. Polyploidy in banana makes breeding a difficult process owing to complexities resulting from parthenocarpy and sterility. Besides, the degree of sterility is particularly high in edible cultivars, breeding of banana is complicated and time consuming Shepherd (1954, 1960). A minimum of two years is required to complete a seed-to-seed crop cycle. Even after thousands of crosses, very few viable seedlings were obtained from a limited percentage of seed set and each plant occupied $6 \mathrm{~m}^{2}$ in the field for evaluation by Rowe (1984).

Stomata size was proportional to ploidy in banana, while stomatal density had the expected complementary relationship as reported by Rowe (1984), Simmonds (1948) and Borges (1971). A number of ploidy levels exist in Musa spp. by Tenkouano et al., (2011). Knowledge of ploidy level in Musa 
accessions is vital for breeding, conservation and tissue culture as they are affected by ploidy Suman et al., (2012). Ploidy level influences fertility of banana. For instance, most triploids are sterile while diploids and tetraploids are fertile by Tenkouano et al., (2011). Banana breeding usually involves the transfer of useful genes from diploids to triploids by carrying out $3 \mathrm{x}$ by $2 \mathrm{x}$ crosses. Such a cross can generate a variety of progeny with ploidy levels ranging from diploid, triploid, tetraploid, aneuploidy and hyperploids progeny by Pillay et al., (2002). Ploidy level of banana determined primarily by morphological characteristics by Pillay et al., (2003), Pillay et al., (2006). The ploidy level is determined by other several methods, of which flow cytometry has screening a large number of accessions by Tenkouano et al., (2011). In Jamaica breeding programme, Smith et al., (1993) reported that the stomatal densities of two month old seedlings were employed to screen the progenies ploidy levels and genomic constitutions. Tetraploids derived from the diploid clone SH-3362 had a mean stomatal length of $26.9 \mu \mathrm{m}$ as against $16.0 \mu \mathrm{m}$ in the diploid.

Currently, the genomic constitution of the new hybrids was assessed by morphological scoring method developed Simmonds and Shepherd (1955) and also referred to the scoring suggested by Simmonds and Shepherd (1987), Singh and Uma (1987, 1996). Flow cytometry enables rapid and precise measurements on whole cells, isolated nuclei or chromosomes in a monodisperse suspension. Van Duren et al., (1996) used this technique to identify the in vitro induced tetraploids of SH-3362 banana clone. Since it involves determination of nuclear DNA, is more in the reliability of ploidy is more in detection by Dolezel (1997). Among the 24 hybrids evaluated by Das (2008), three were found to be pentaploids as confirmed by flowcytometry.

\section{Materials and Methods}

The present study was taken up at the College orchard, Horticultural College and Research Institute, Tamil Nadu Agricultural University, Coimbatore, Tamil Nadu.

\section{Assessment of ploidy of hybrids}

The ploidy status of the hybrids was assessed by the estimation of stomatal density and size at cellular level as postulated by Sathiamoorthy (1973). Ploidy levels of hybrids obtained from different cross combinations is a must in banana breeding because of potential production of diploid, triploid, tetraploid, hyperploid and aneuploid hybrids. Ploidy levels are estimated by phenotypic appearance and confirmed lither by root tip mitosis or stomatal density, size and number of chloroplast per guard cell pair. Sathiyamoorthy (1973) and Vandenhout et al., (1995) classified banana clones diploids, triploids and tetraploids based on stomatal density and stomatal size, respectively.

\section{Stomatal density}

The sample for stomatal study was taken from the centre portion of the third leaf. The sample leaves were cut into one centimetre ${ }^{2}$ bits and boiled for two minutes in water and then transferred to 70 per cent ethanol, where it was kept for 24 hours to remove the chlorophyll. The sample was then washed with water and boiled in 70 per cent lactic acid for five minutes to soften the tissues. The treated sample bit was kept over a clean slide with the upper surface of lamina bit in contact with the slide. The tissues were gently scrapped with a sharp blade and the intervening fibers were removed carefully with a pointed needle, till the upper epidermis alone was in contact with the slide. The material was gently washed and mounted in glycerin and sealed with a cover slip and 
examined under microscope of $45 \mathrm{x}$ magnifications by Sathiyamoorthy (1973). The number of stomata per microscopic field $\left(0.152 \mathrm{~mm}^{2}\right)$ was counted at least at ten different fields and the mean was arrived. The result was expressed as number of stomata per $\mathrm{mm}^{2}$. Besides, the length and breadth of the stomata were also measured by using ocular micrometer (Plate 1 and 2). The size of the stomata was calculated by multiplying the length and breadth and was expressed in $\mu \mathrm{m}^{2}$. The stomatal density and size of the hybrid seedlings were used to group the hybrids into diploids, triploids and tetraploids as indicated below:

\section{Analysis of genome}

The genomic constitution of the new hybrids was assessed by morphological scoring method (Table 1) by Simmonds and Shepherd (1955) and modified scoring (Table 2) by Singh and Uma (1996).

Young cigar leaves of selected hybrids were analysed for their ploidy level by measuring the size of the nuclear genome by this method. The cigar leaves were cut using sharp sterile blade up to 15-20 centimetres length from top, cleaned gently with sterile distilled water and wrapped with partially wetted sterilized whatman No.3 filter paper. The samples were then packed in zipped polyethylene cover and sent to the Laboratory of Molecular Cytogenetics and Cytometry, Czech Republic for ploidy analysis by Dolezel (1997). Flow cytometry ploidy assay involved preparation of suspensions of intact nuclei from small amounts of leaf tissue and the analysis of fluorescence intensity after staining. Relative fluorescence intensity of stained nuclei was analysed using a partec ploidy analyser with a mercury arc lamp. The distribution of fluorescence intensities (relative DNA content) obtained after flow cytometric analyses are usually given as channel number.
The ploidy screening, the instrument was calibrated using reference (standard) diploid (2x) with its peak set and other hand was used as the reference tetraploid $(4 x)$ with its peak set and other reference triploid (3x) with its peak set. The peaks of the unknown samples were determined by examining the position of their peaks relative to the reference accessions. Diploid banana $(2 x)$ nuclei were included in every sample as an internal reference standard.

\section{Results and Discussion}

Success of conventional breeding in banana is very limited due to sterility, parthenocarpy and varying ploidy levels. Commercial bananas are mostly triploids and are vegetatively parthenocarpic. Diploids are not suitable because of their reduced fruit size and vigour by Simmonds (1962).

Genome and ploidy assessment based on morphological characters

Among the 24 of phase-I hybrids scored for genome assessment, six were diploid (AA and $A B$ ), five triploid ( $A A A$ and $A A B$ ), ten tetraploid (AABB) and three pentaploids (AAABB) were recorded (Table-3 and 4). Out of 24 hybrids, one triploid, three tetraploid and three pentaploids were (Table 1) confirmed by Flow-cytometry test (Fig. 3, 4 and 5), which is indicated in * mark in the end of the table 3. Among the 19 of Phase-II hybrids evaluated, one diploid $(\mathrm{AB})$, four triploids $(\mathrm{AAB})$ and fourteen tetraploids (AABB) were identified.

\section{Assessment of ploidy status by stomatal characters}

Ploidy levels of the phase I and II hybrids were studied through morphological scoring Simmonds and Shepherd (1955) and Singh et al., (1993) and stomatal density. Among the 24 of phase-I hybrids scored for genome 
assessment, six were diploid ( $\mathrm{AA}$ and $\mathrm{AB}$ ), five triploid (AAA and $\mathrm{AAB}$ ), ten tetraploid $(\mathrm{AABB})$ and three pentaploids (AAABB) were recorded (Table- 3 and 4). Among the 19 hybrids evaluated, one diploid $(\mathrm{AB})$, four triploids (AAB) and fourteen tetraploids (AABB) were identified (Table 5 and 6 and Fig. 2).

The various indirect methods of determining banana ploidy level, for example by estimating stomatal size and density by Vandenhout et al., (1995) or measurement of pollen grain sizes by Tenkouano et al., (1998). The ploidy status of newly developed hybrids was assessed based on microscopic measurements of density and size of stomata in the leaves of the respective hybrids. Among the 24 hybrids scored for genome assessment, with reference to the stomatal density, all confirmed with morphological and flow cytometry tests already conducted. However, the genome, $\mathrm{H}$ 511, recorded a stomatal density of 1711.29 which is below the level of tetraploid, confirmed through flow cytometry. Among the diploid which was, the stomatal density varied from 50.79 to $85.02 / \mathrm{mm}^{2}$ while in triploid, it ranged from 32.51 and $47.26 / \mathrm{mm}^{2}$ in tetraploids, it ranged from 13.15 to $17.42 / \mathrm{mm}^{2}$ but in pentaploids, the range was from 5.02 to $7.89 / \mathrm{mm}^{2}$ (Table 4 and Fig. 1). The stomatal density decreased with the increase in ploidy level. The mean stomatal length, breadth and size in hybrids were $28.05 \mu \mathrm{m}, \quad 25.91 \mu \mathrm{m}$ and $726.89 \mu \mathrm{m}^{2}$ respectively for diploids; $36.79 \mu \mathrm{m}, 33.95 \mu \mathrm{m}$ and $1252.43 \mu \mathrm{m}^{2}$ respectively for triploids; $46.02 \mu \mathrm{m}, \quad 41.82 \mu \mathrm{m}$ and $1921.66 \mu \mathrm{m}^{2}$ respectively for tetraploids and $51.74 \mu \mathrm{m}$, $44.64 \mu \mathrm{m}$ and $2311.60 \mu \mathrm{m}^{2}$ respectively for pentaploids (Table 3 and 4 and Fig. 2). Based on stomatal density, length, breadth and size, the hybrids were grouped into diploids, triploids and tetraploids. Similarly, phase-II hybrids (Table 5 and 6), the diploid H-03-06 recorded a stomatal density of $55.20 / \mathrm{mm}^{2}$ and in triploids, it ranged from 32.89 to $47.14 / \mathrm{mm}^{2}$. Among the tetraploids, the hybrid H-03-05 registered the minimum number (12.15 stomata $/ \mathrm{mm}^{2}$ ), while the hybrid H-0219 registered the maximum 29.20 stomata $/ \mathrm{mm}^{2}$. The stomatal size varied significantly with ploidy levels and a minimum of $493.74 \mu \mathrm{m}^{2}$ was recorded by the diploid hybrid $\mathrm{H}-03-06$, while the maximum $2318.42 \mu \mathrm{m}^{2}$ by the tetraploid hybrid $\mathrm{H}-02-19$ (Table 5 and 6). Reliability of ploidy determination using stomatal measurements by correlating stomatal traits with chromosome counts in root tips of the hybrids 'Obino 1' Ewai $\times$ Calcutta 4 was carried out by Vandenhout et al., (1995). Size and densities of stomata, which are negatively correlated, varied according to ploidy level. Diploid hybrids had an average of 29 stomata/ $\mathrm{mm}^{2}$ with an average size (length $x$ width) of $1250 \mu \mathrm{m}^{2}$, while tetraploids had an average of 15 stomata $/ \mathrm{mm}^{2}$ with an average size of $1840 \mu \mathrm{m}^{2}$. In a similar observation reported by Elain Apshara (2000) observed stomatal densities namely $43.52 / \mathrm{mm}^{2}$, $31.08 / \mathrm{mm}^{2}, 17.27 / \mathrm{mm}^{2}$ and $10.50 / \mathrm{mm}^{2}$ for diploid, triploid, tetraploid and pentaploid hybrids, respectively.

Assessment of ploidy by using flowcytometry

The hybrids viz., H 504, H 511, H 534, H 537, $\mathrm{H}$ 540, H571 and H 573, which were found deviating from the scale and score, were referred to Dr. Jaroslav Dolezol, Laboratory of Molecular cytogenetics and cytometry, Institute of Experimental Botany, Czech Republic for flow cytometry analysis to fix the ploidy levels. The result of flow-cytometry analysis revealed that one triploid, three tetraploid and three pentaploid progenies (Table 4). The ploidy of individual plant was estimated based on the ratio of peaks corresponding to G1 nuclei of Musa sample and reference standard (2x) (Fig. 3, 4 and 5). 


\section{Stomatal density}

\begin{tabular}{|l|c|c|}
\multicolumn{1}{|c|}{ Ploidy } & $\begin{array}{c}\text { Stomatal density } \\
(\text { No. of stomata/mm }\end{array}$ & Stomatal size $\left(\boldsymbol{\mu m}^{\mathbf{2}}\right)$ \\
\hline Diploids & $40.00-50.00$ & 1250.00 \\
\hline Triploids & $30.00-40.00$ & $1250-1840$ \\
\hline Tetraploids & $9.00-15.20$ & 1840.00 \\
& Sathiamoorthy (1973) & Vandenhout et. al., (1995) \\
\hline
\end{tabular}

Table.1 Taxonomic scoring of banana cultivars by Simmonds and Shepherd (1955)

\begin{tabular}{|c|c|c|c|}
\hline S. No & Character & M. acuminata & M. balbisiana \\
\hline 1 & Pseudostem colour & $\begin{array}{l}\text { More or less heavily marked with black } \\
\text { or brown blotches }\end{array}$ & Blotches slight or absent \\
\hline 2 & Petiolar canal & $\begin{array}{l}\text { Margin erect or spreading with scarious } \\
\text { wings below, not clasping pseudostem }\end{array}$ & $\begin{array}{l}\text { Margins not winged below, clasping } \\
\text { pseudostem }\end{array}$ \\
\hline 3 & Peduncle & Usually downy or hairy, short & Glabrous \\
\hline 4 & Pedicel & Short & Long \\
\hline 5 & Ovules & Two regular rows in each locule & Four irregular rows in each locule \\
\hline 6 & Bract shoulder ratio & Usually high $(<0.28)$ & Usually low (>0.28) \\
\hline 7 & Bract curling & Bract roll & Bracts lift but do not roll \\
\hline 8 & Bract shape & Lanceolate or narrowly ovate & Broadly ovate, not tapering sharply \\
\hline 9 & Bract apex & Acute & Obtuse \\
\hline 10 & Bract colour & $\begin{array}{l}\text { Red, dull purple or yellow outside, } \\
\text { pink, dull purple or yellow inside }\end{array}$ & $\begin{array}{l}\text { Distinctive, brownish purple } \\
\text { outside: bright crimson inside }\end{array}$ \\
\hline 11 & Colour fading & Inside bract colour fades to yellow base & $\begin{array}{l}\text { Inside bract colour is continuous till } \\
\text { base }\end{array}$ \\
\hline 12 & Bract scars & Prominent & Scarcely prominent \\
\hline 13 & Free tepal of male flower & Variably corrugated below the tip & Rarely corrugated \\
\hline 14 & Male flower colour & Creamy white & Variably flushed with pink \\
\hline 15 & Stigma colour & Orange or rich yellow or pale pink & Cream pale yellow \\
\hline
\end{tabular}

Table.2 Modified scoring by Singh and Uma (1996)

\begin{tabular}{|l|c|c|}
\hline \multirow{2}{*}{ Genomes } & \multicolumn{2}{|c|}{ Score card } \\
\hline AA/AAA & Simmonds and Shepherd (1955). & Singh and Uma (1996). \\
\hline AAB & $15-23$ & $15-25$ \\
\hline AB & $24-46$ & $26-45$ \\
\hline ABB & 49 & $46-49$ \\
\hline ABBB & $59-63$ & $59-65$ \\
\hline BB/BBB & 67 & $66-69$ \\
\hline
\end{tabular}

Flow- cytometry analysis 
Int.J.Curr.Microbiol.App.Sci (2018) 7(8): 3251-3264

Table.3 Genome assessment of banana hybrids under phase I evaluation

\begin{tabular}{|c|c|c|c|c|c|c|}
\hline S.N & Hybrids & Parentage & Genome & Scoring & $\begin{array}{c}\text { Chromoso } \\
\text { me no. }\end{array}$ & Ploidy \\
\hline 1 & H $504 * * *$ & H-03-09 x PL & AAABB & 51.0 & 55 & $5 X$ \\
\hline 2 & H 508 & ANK $x$ PL & AA & 21.0 & 22 & $2 \mathrm{X}$ \\
\hline 3 & H $511^{* *}$ & H-02-34 x Ykm-5 & $\mathrm{AABB}$ & 56.0 & 44 & $4 \mathrm{X}$ \\
\hline 4 & Н 515 & Mano x ANK & AAA & 22.0 & 33 & $3 \mathrm{X}$ \\
\hline 5 & Н 516 & ANK x PL & AA & 23.0 & 22 & $2 \mathrm{X}$ \\
\hline 6 & H 529 & H-03-16 x ANK & AABB & 52.0 & 44 & $4 \mathrm{X}$ \\
\hline 7 & H 530 & $\mathrm{H}-03-13$ (OP) & AABB & 53.0 & 44 & $4 \mathrm{X}$ \\
\hline 8 & Н 531 & Poovan x PL & $\mathrm{AAB}$ & 28.0 & 33 & $3 \mathrm{X}$ \\
\hline 9 & Н 532 & H-201 x Mano & $\mathrm{AAB}$ & 29.0 & 33 & $3 X$ \\
\hline 10 & H 534* & H-03- 13 x Rose & $\mathrm{AAB}$ & 38.0 & 33 & $3 \mathrm{X}$ \\
\hline 11 & H $537 * *$ & $(\mathrm{H}-201 \mathrm{x}$ PK) x Rose & AABB & 52.0 & 44 & $4 \mathrm{X}$ \\
\hline 12 & H $540 * * *$ & $(\mathrm{H}-201 \times \mathrm{PK}) \times$ Rose & AAABB & 54.0 & 55 & $5 \mathrm{X}$ \\
\hline 13 & Н 542 & H-02-34 x ANK & $\mathrm{AABB}$ & 55.0 & 44 & $4 \mathrm{X}$ \\
\hline 14 & Н 547 & $\mathrm{H}-02-23(\mathrm{OP})$ & $\mathrm{AABB}$ & 53.0 & 44 & $4 \mathrm{X}$ \\
\hline 15 & Н 548 & $\mathrm{H}-02-23(\mathrm{OP})$ & $\mathrm{AABB}$ & 56.0 & 44 & $4 \mathrm{X}$ \\
\hline 16 & H 556 & H-04-06 x Ykm-5 & $\mathrm{AABB}$ & 59.0 & 44 & $4 \mathrm{X}$ \\
\hline 17 & Н 563 & H-201 x PL & $\mathrm{AB}$ & 44.0 & 22 & $2 \mathrm{X}$ \\
\hline 18 & Н 564 & H-201 x PL & $\mathrm{AB}$ & 46.0 & 22 & $2 \mathrm{X}$ \\
\hline 19 & H $571 * *$ & $\mathrm{H}-04-05$ x Ykm-5 & $\mathrm{AABB}$ & 63.0 & 44 & $4 \mathrm{X}$ \\
\hline 20 & Н 572 & H-03-35 (OP) & $\mathrm{AAB}$ & 28.0 & 33 & $3 \mathrm{X}$ \\
\hline 21 & H $573 * * *$ & H-03-12 x Rose & AAABB & 61.0 & 55 & $5 \mathrm{X}$ \\
\hline 22 & Н 576 & H-201(OP) & $\mathrm{AB}$ & 46.0 & 22 & $2 \mathrm{X}$ \\
\hline 23 & Н 579 & Mano x Rose & $\mathrm{AA}$ & 25.0 & 22 & $2 \mathrm{X}$ \\
\hline 24 & Н 589 & H-03-19 (OP) & AABB & 57.0 & 44 & $4 \mathrm{X}$ \\
\hline
\end{tabular}

PL- Pisang Lilin; ANK - Anaikomban; PK-Peykunnan; OP- Open Pollinated; Mano- Manoranjitham AA/ AAA-15-25; AAB-26-45; AB-46-49; ABB-59-65; ABBB-66-69

(* Triploid, ** Tetraploid, *** Pentaploid- Flow cytometry tested) 
Table.4 Assessment of ploidy in phase I hybrids by stomatal characters

\begin{tabular}{|c|c|c|c|c|c|c|c|c|}
\hline $\begin{array}{l}\text { S. } \\
\text { No }\end{array}$ & Hybrids & Parentage & Genome & Ploidy & $\begin{array}{c}\text { Stomatal } \\
\text { density } \\
\left(\text { no. } / \mathbf{m m}^{2)}\right.\end{array}$ & $\begin{array}{c}\text { Stomatal } \\
\text { length }(\mu \mathrm{m})\end{array}$ & $\begin{array}{c}\text { Stomatal } \\
\text { Breadth } \\
(\mu \mathrm{m})\end{array}$ & $\begin{array}{l}\text { Stomatal } \\
\operatorname{size}\left(\mu \mathrm{m}^{2}\right)\end{array}$ \\
\hline 1 & H-504 & H-03-09 x PL & AAABB & $5 X$ & 6.16 & 51.44 & 45.62 & 2346.69 \\
\hline 2 & H-508 & ANK $\times$ PL & AA & $2 X$ & 85.02 & 25.70 & 26.4 & 678.48 \\
\hline 3 & H-511 & H-02-34 x Ykm\#5 & AABB & $4 X$ & 20.13 & 45.72 & 37.43 & 1711.29 \\
\hline 4 & H-515 & Mano. x ANK & AAA & $3 X$ & 34.59 & 37.20 & 34.30 & 1275.96 \\
\hline 5 & H-516 & ANK x PL & AA & $2 X$ & 83.60 & 25.80 & 25.40 & 655.32 \\
\hline 6 & Н 529 & H-03-16 x ANK & $\mathrm{AABB}$ & $4 X$ & 21.47 & 45.35 & 41.98 & 1903.79 \\
\hline 7 & Н 530 & H-03-13 (OP) & $\mathrm{AABB}$ & $4 X$ & 24.27 & 45.66 & 42.20 & 1926.85 \\
\hline 8 & H-531 & Poovan x PL & $\mathrm{AAB}$ & $3 X$ & 37.26 & 37.20 & 35.25 & 1311.30 \\
\hline 9 & H-532 & H-201 x Mano. & $\mathrm{AAB}$ & $3 X$ & 35.00 & 37.10 & 34.60 & 1283.66 \\
\hline 10 & H-534 & H-03-13 x Rose & $\mathrm{AAB}$ & $3 X$ & 30.08 & 38.84 & 35.41 & 1375.32 \\
\hline 11 & H-537 & $(\mathrm{H}-201 \times \mathrm{PK}) \times$ Rose & $\mathrm{AABB}$ & $4 X$ & 22.51 & 49.19 & 46.55 & 2289.79 \\
\hline 12 & H-540 & $(\mathrm{H}-201 \times \mathrm{PK}) \times$ Rose & AAABB & $5 X$ & 5.02 & 56.25 & 44.65 & 2511.56 \\
\hline 13 & Н 542 & H-02-34 x ANK & $\mathrm{AABB}$ & $4 X$ & 16.44 & 46.20 & 41.40 & 1912.68 \\
\hline 14 & H-547 & $\mathrm{H}-02-23(\mathrm{OP})$ & $\mathrm{AABB}$ & $4 X$ & 23.39 & 46.35 & 41.76 & 1935.38 \\
\hline 15 & H-548 & H-02-23(OP) & AABB & $4 X$ & 28.45 & 45.27 & 42.48 & 1923.07 \\
\hline 16 & H-556 & $\mathrm{H}-04-06$ x Ykm\#5 & $\mathrm{AABB}$ & $4 X$ & 13.89 & 44.73 & 41.98 & 1877.77 \\
\hline 17 & Н 563 & H-201 x PL & $\mathrm{AB}$ & $2 X$ & 50.79 & 29.67 & 24.49 & 726.62 \\
\hline 18 & Н 564 & H-201 x PL & $\mathrm{AB}$ & $2 \mathrm{X}$ & 51.45 & 28.95 & 26.55 & 768.62 \\
\hline 19 & Н 571 & H-04-05 x Ykm\#5 & $\mathrm{AABB}$ & $4 X$ & 25.08 & 44.47 & 41.92 & 1864.18 \\
\hline 20 & H 572 & H-03-35 (OP) & $\mathrm{AAB}$ & $3 X$ & 32.51 & 38.60 & 36.20 & 1397.32 \\
\hline 21 & H-573 & H-03-12 x Rose & $\mathrm{AAABB}$ & $5 X$ & 7.89 & 47.54 & 43.68 & 2076.55 \\
\hline 22 & Н 576 & H-201 (OP) & $\mathrm{AB}$ & $2 X$ & 73.39 & 26.20 & 25.90 & 678.58 \\
\hline 23 & Н 579 & Mano. x Rose & $\mathrm{AA}$ & $2 X$ & 77.63 & 31.95 & 26.72 & 853.70 \\
\hline 24 & Н 589 & H-03-19 (OP) & $\mathrm{AABB}$ & $4 X$ & 28.95 & 47.26 & 39.53 & 1868.19 \\
\hline
\end{tabular}

PL - Pisang Lilin; ANK - Anaikomban; PK- Peykunnan; OP- Open pollinated; Mano - Manoranjitham

Table.5 Genome and ploidy assessment in phase II hybrids through morphological scoring (Sucker to Harvest)

\begin{tabular}{|c|c|c|c|c|c|}
\hline S.N & Hybrids & Parentage & Genome & Mark scored & Ploidy \\
\hline 1 & H-02-19 & KAR x RED & AABB & 60 & $4 X$ \\
\hline 2 & H-02-23 & KAR x RED & AABB & 59 & $4 X$ \\
\hline 3 & H-02-26 & KAR x RED & AABB & 63 & $4 X$ \\
\hline 4 & H-02-34 & KAR x RED & AABB & 62 & $4 X$ \\
\hline 5 & H-03-05 & Peykunnan (OP) & $\mathrm{AABB}$ & 59 & $4 X$ \\
\hline 6 & H-03-06 & H-02-32 x PL & $\mathrm{AB}$ & 49 & $2 X$ \\
\hline 7 & H-03-13 & Peykunnan x EV & $\mathrm{AABB}$ & 56 & $4 X$ \\
\hline 8 & H-03-16 & Peykunnan x PL & AABB & 62 & $4 X$ \\
\hline 9 & H-03-17 & Peykunnan x PL & $\mathrm{AABB}$ & 58 & $4 X$ \\
\hline 10 & H-03-19 & Peykunnan x EV & $\mathrm{AABB}$ & 60 & $4 X$ \\
\hline 11 & H-04-05 & $\mathrm{H}-02-32 \times \mathrm{PL}$ & $\mathrm{AABB}$ & 47 & $4 X$ \\
\hline 12 & H-04-06 & $\mathrm{H}-02-32 \times \mathrm{PL}$ & $\mathrm{AABB}$ & 35 & $4 X$ \\
\hline 13 & H-04-10 & Peykunnan (OP) & $\mathrm{AAB}$ & 30 & $3 X$ \\
\hline 14 & H-04-12 & Pisang Saba x PL & $\mathrm{AABB}$ & 62 & $4 X$ \\
\hline 15 & H-04-21 & $\mathrm{H}-02-10 \times \mathrm{PL}$ & $\mathrm{AAB}$ & 44 & $3 X$ \\
\hline 16 & H-04-24 & Peykunnan (OP) & $\mathrm{AABB}$ & 61 & $4 X$ \\
\hline 17 & NPH-02-01 & H 201 x ANK & $\mathrm{AAB}$ & 42 & $3 X$ \\
\hline 18 & H-510 & Poovan (OP) & $\mathrm{AABB}$ & 61 & $4 X$ \\
\hline 19 & H-531 & Poovan $x$ PL & $\mathrm{AAB}$ & 28 & $3 X$ \\
\hline
\end{tabular}

AA/ AAA-15-25; AAB-26-45; AB-46-49; ABB-59-65; ABBB-66-69 ANK - Anaikomban; EV - Erachivazhai; PL - Pisang Lilin; OP- Open pollinated; KAR-Karpooravalli; RED- Red banana 
Table.6 Assessment of ploidy in phase II hybrids by stomatal characters

\begin{tabular}{|c|c|c|c|c|c|c|c|}
\hline S.N & Hybrids & Parentage & Genome & $\begin{array}{c}\text { Stomatal } \\
\text { density } \\
\left(\mathbf{m m}^{2}\right)\end{array}$ & $\begin{array}{c}\text { Stomatal } \\
\text { length } \\
(\mu \mathrm{m})\end{array}$ & $\begin{array}{c}\text { Stomatal } \\
\text { Breadth } \\
(\mu \mathrm{m})\end{array}$ & $\begin{array}{c}\text { Stomatal } \\
\text { size } \\
\left(\mu \mathrm{m}^{2}\right)\end{array}$ \\
\hline 1 & H-02-19 & KAR x RED & AABB & 29.20 & 48.20 & 48.10 & 2318.42 \\
\hline 2 & H- $02-23$ & KAR x RED & $\mathrm{AABB}$ & 22.10 & 35.00 & 35.00 & 1225.00 \\
\hline 3 & H-02-26 & KAR x RED & $\mathrm{AABB}$ & 28.15 & 41.00 & 41.00 & 1681.00 \\
\hline 4 & $\mathrm{H}-02-34$ & KAR $\times$ RED & AABB & 23.00 & 31.80 & 31.60 & 1004.88 \\
\hline 5 & H-03-05 & $\begin{array}{l}\text { Peykunnan } \\
\text { (OP) }\end{array}$ & $\mathrm{AABB}$ & 12.15 & 42.20 & 37.70 & 1590.94 \\
\hline 6 & H-03-06 & $\mathrm{H}-02-32 \times \mathrm{PL}$ & $\mathrm{AB}$ & 55.20 & 23.40 & 21.10 & 493.74 \\
\hline 7 & H-03-13 & $\begin{array}{l}\text { Peykunnan x } \\
\text { EV }\end{array}$ & $\mathrm{AABB}$ & 19.12 & 43.10 & 41.05 & 1795.12 \\
\hline 8 & H-03-16 & $\begin{array}{l}\text { Peykunnan x } \\
\text { PL }\end{array}$ & $\mathrm{AABB}$ & 18.60 & 41.90 & 35.15 & 1472.79 \\
\hline 9 & H-03-17 & $\begin{array}{l}\text { Peykunnan } \mathrm{x} \\
\text { PL }\end{array}$ & $\mathrm{AABB}$ & 12.70 & 38.45 & 31.86 & 1225.02 \\
\hline 10 & H-03-19 & $\begin{array}{l}\text { Peykunnan } x \\
\text { EV }\end{array}$ & $\mathrm{AABB}$ & 20.70 & 39.55 & 34.94 & 1381.88 \\
\hline 11 & H-04-05 & $\mathrm{H}-02-32 \times \mathrm{PL}$ & AABB & 13.15 & 44.10 & 41.65 & 1836.77 \\
\hline 12 & H-04-06 & $\mathrm{H}-02-32 \times \mathrm{PL}$ & $\mathrm{AABB}$ & 16.44 & 39.60 & 36.75 & 1455.30 \\
\hline 13 & H-04-10 & $\begin{array}{l}\text { Peykunnan } \\
\text { (OP) }\end{array}$ & $\mathrm{AAB}$ & 32.89 & 38.60 & 31.86 & 1229.80 \\
\hline 14 & H-04-12 & $\begin{array}{c}\text { Pisang Saba } x \\
\text { PL }\end{array}$ & $\mathrm{AABB}$ & 13.15 & 42.58 & 34.60 & 1473.27 \\
\hline 15 & H-04-21 & $\mathrm{H}-02-10 \times \mathrm{PL}$ & $\mathrm{AAB}$ & 47.14 & 38.45 & 32.77 & 1260.01 \\
\hline 16 & H-04-24 & $\begin{array}{l}\text { Peykunnan } \\
\text { (OP) }\end{array}$ & $\mathrm{AABB}$ & 13.18 & 39.55 & 36.90 & 1459.40 \\
\hline 17 & $\begin{array}{l}\text { NPH-02- } \\
01\end{array}$ & H 201 x ANK & $\mathrm{AAB}$ & 36.20 & 25.55 & 23.60 & 602.98 \\
\hline 18 & H-510 & Poovan (OP) & AABB & 23.28 & 39.35 & 36.70 & 1444.15 \\
\hline 19 & H-531 & Poovan x PL & $\mathrm{AAB}$ & 37.00 & 37.20 & 35.25 & 1311.30 \\
\hline \multicolumn{4}{|c|}{ SEd } & 0.913 & 0.589 & 0.542 & 26.166 \\
\hline \multicolumn{4}{|c|}{$\mathrm{CD}(.05 \%)$} & 1.853 & 1.194 & 1.099 & 53.073 \\
\hline \multicolumn{4}{|c|}{$\mathrm{CD}(.01 \%)$} & 2.484 & 1.601 & 1.474 & 71.165 \\
\hline
\end{tabular}

ANK - Anaikomban; EV - Erachivazhai; PL - Pisang Lilin; OP- Open pollinated; KAR-Karpooravalli; RED- Red banana 


\section{Plate 1. Stomatal variation in Banana Hybrids}

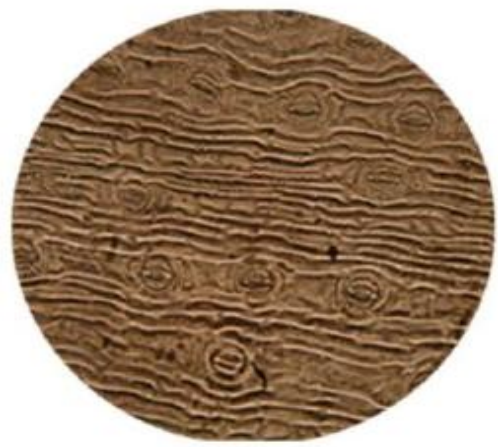

H 516(AA)

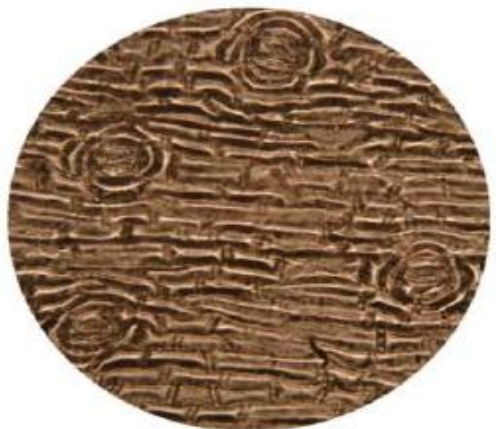

H-537(AABB)

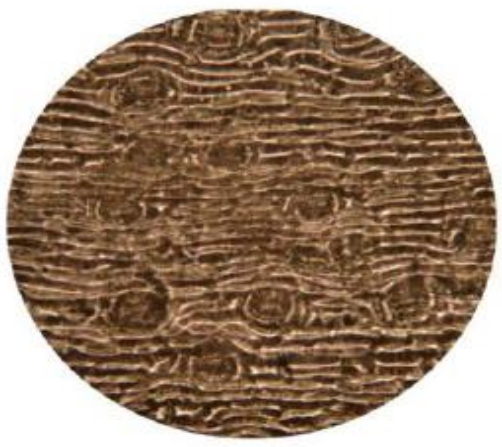

H 515(AAA)

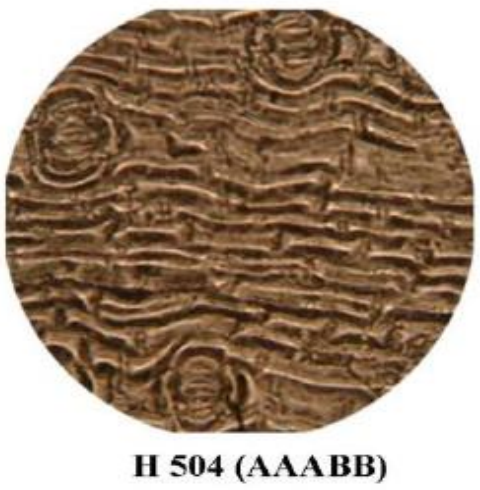

Plate 2. Palynological studies of Banana bybrids

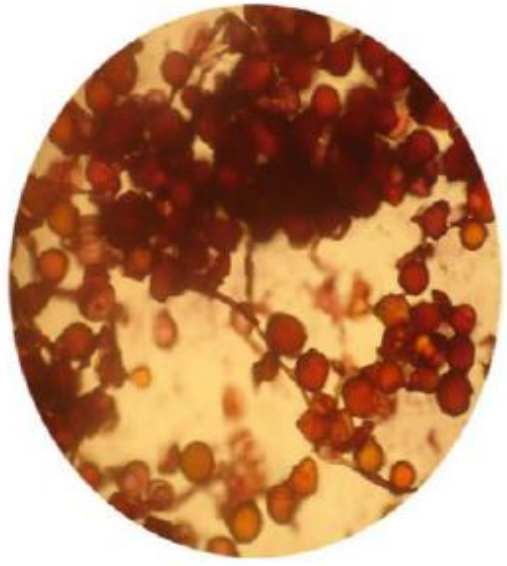

H 572

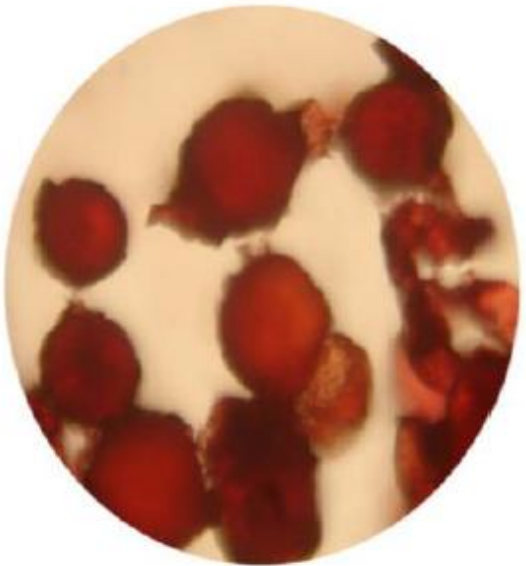

H 537

Highly pollineferous 


\section{Fig 1. Stomatol variation in phase I hybrids}

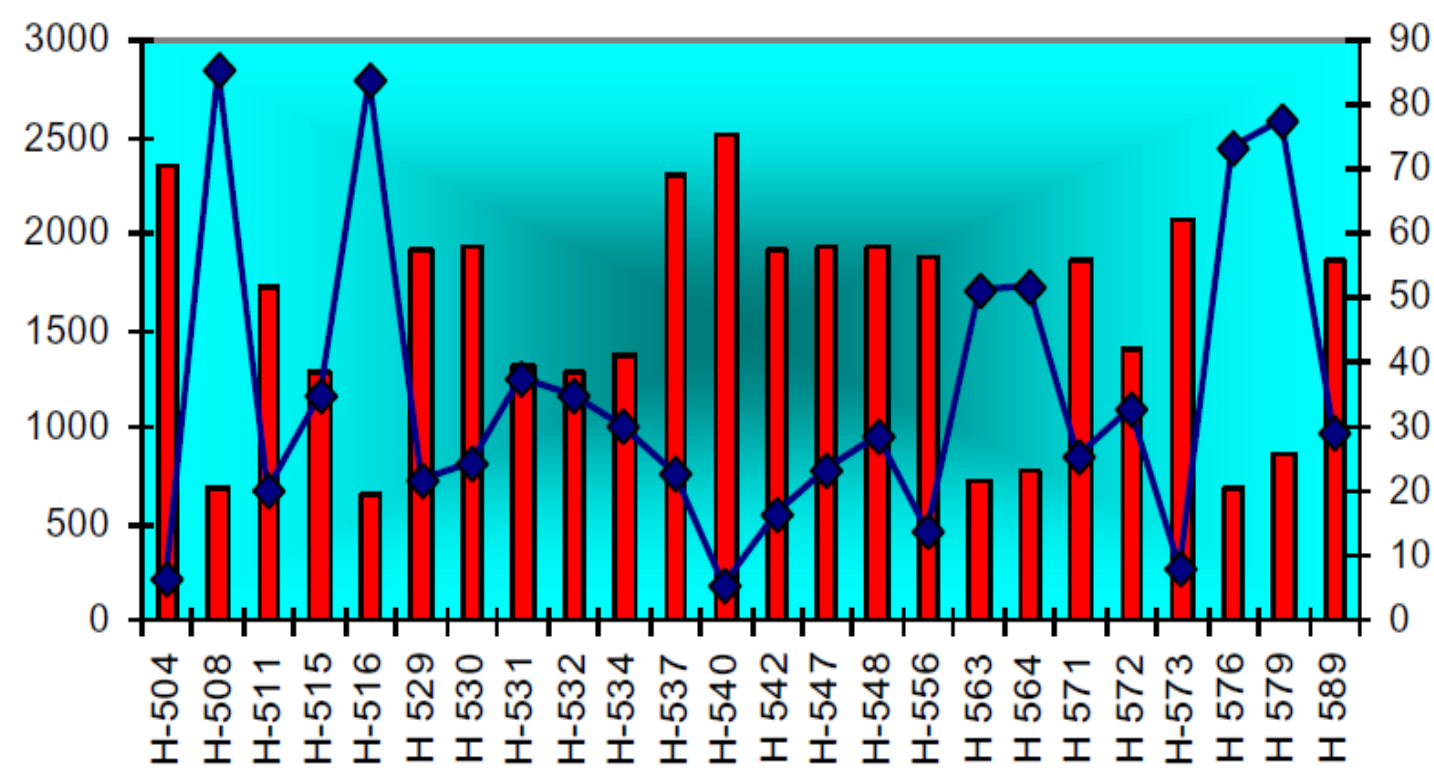

Hybrids

\section{Stomatol size $(\mu \mathrm{m} 2) \quad \sim$ Stmatol density (no./mm2)}

Fig 2. Stomatal variation in phase II hybrids

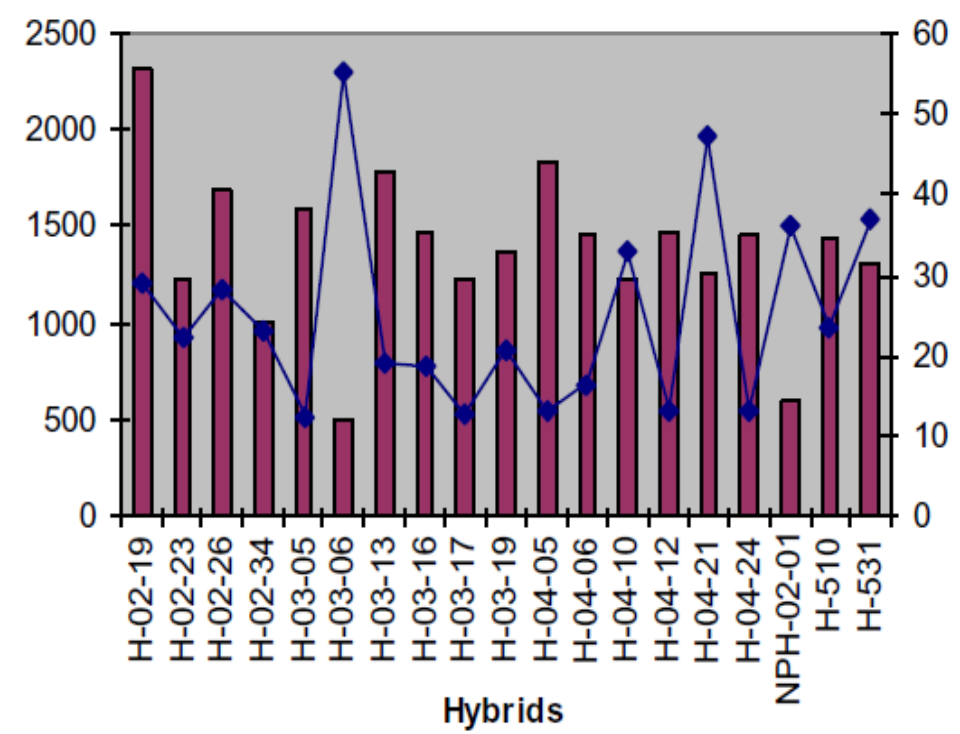


Fig:3 H 504 (AABBB)

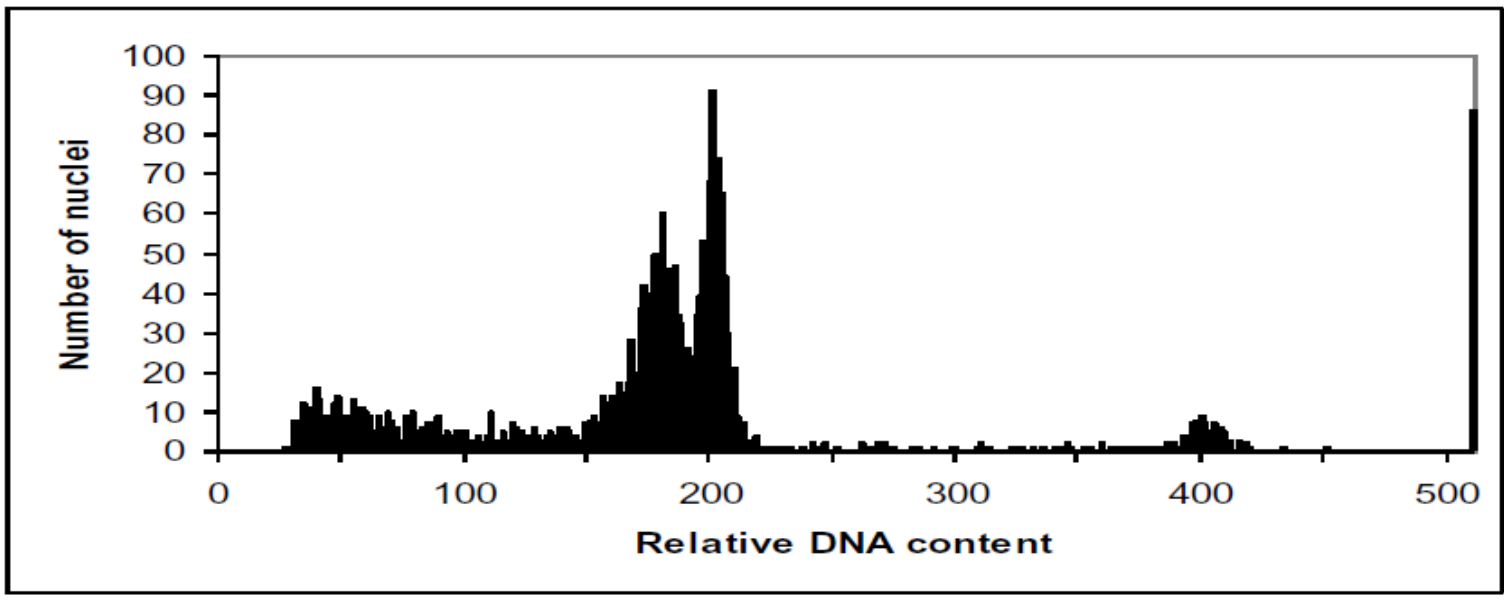

Fig: 4

\section{H511 (AABB)}

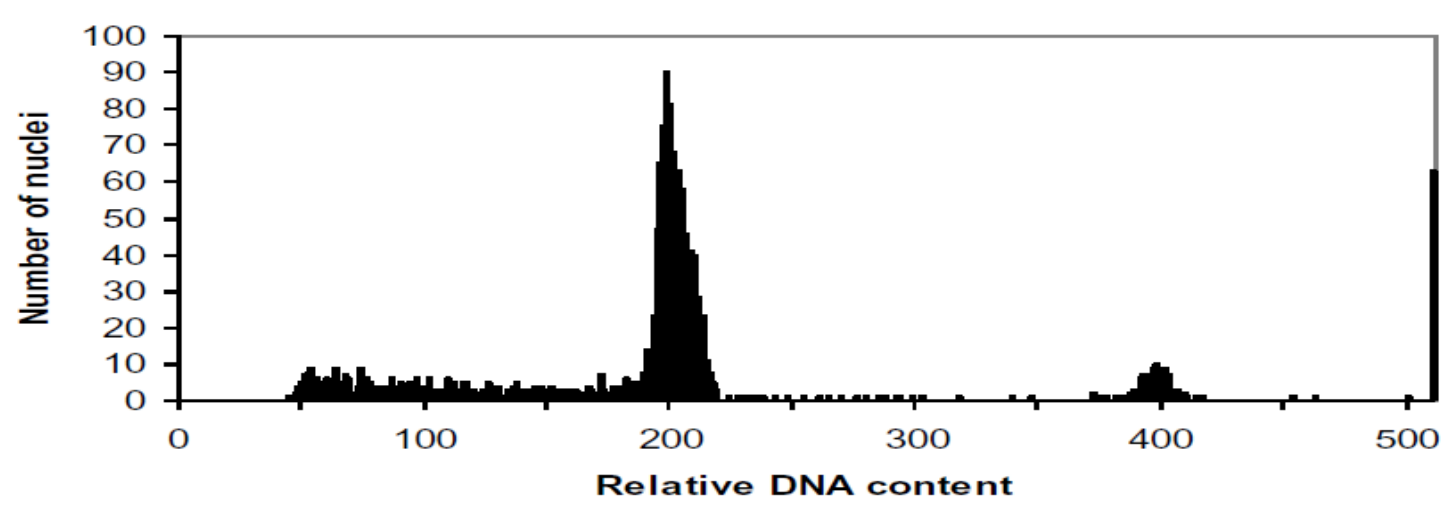

Fig:5

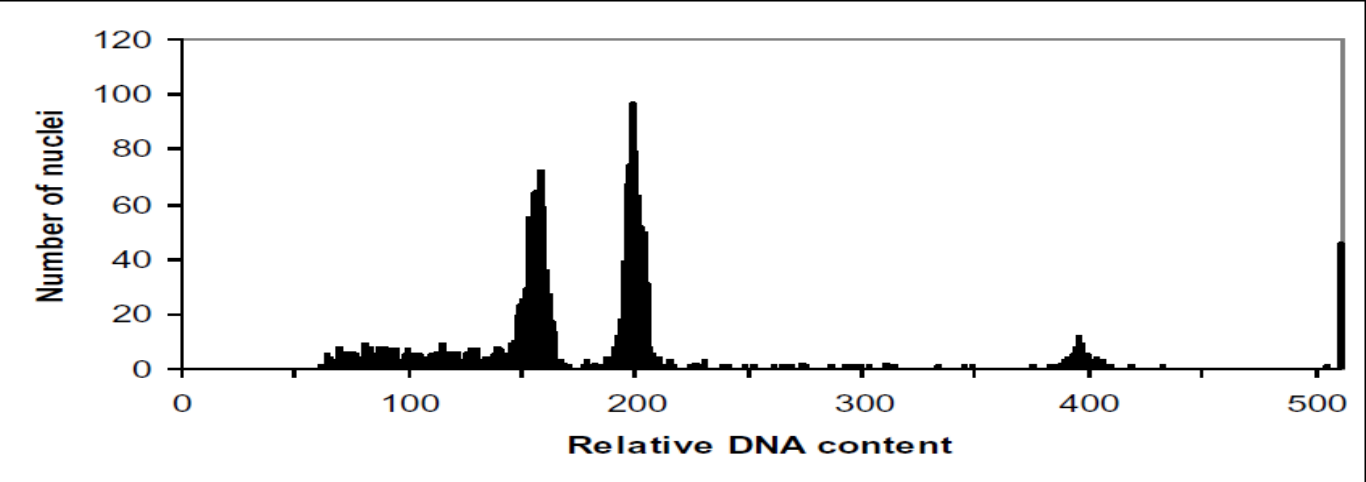

Fig: 3, 4 and 5. Flow cytometry analysis of selective banana hybrids for ploidy confirmation 
Banana taxonomists have always assigned ploidy levels to different accessions on the basis of morphological traits such as leaf orientation, and biochemical aspects by Mustafa (2013). The ploidy of plants with large chromosomes can easily be determined by chromosome counting but bananas present a challenge due to its small chromosomes which are always hard to spread out during squash preparations by Dolezel et al.,(1998), Pillay and Tenkouano (2011). Flow cytometry is a user-friendly technique, considering the fact that it is faster and reproducible for screening large number of accessions.

\section{Ploidy and parthenocarpy assessment of hybrids}

Ploidy level of banana hybrids was fixed through morphological scoring as described by Simmonds (1952) and Singh et al., (2001). Besides, stomatal density and flow cytometry analysis of nuclear DNA by Dolezel et al., (1998) were the other tools used in recent years. Among the three methods, flow cytometry analysis is considered as the recent and reliable because, it is precise and rapid method when other methods were inconclusive. Precision is more because of the analysis of the nuclear DNA, which is not affected by the environmental factors. In the present investigation, ploidy was fixed using stomatal density, morphological scoring and flowcytometry. Among the 24 hybrids, in phase-I, 6 were found to be diploid ( $\mathrm{AA}$ and $\mathrm{AB}$ ), 5 triploids (AAA and AAB), 10 tetraploids (AABB) and 3 pentaploids (AAABB) (Table 3 and 4). The pentaploid hybrids obtained in this investigation were resulted from the cross between tetraploids (AABB) as female and diploids (AA) as male parent. Classification based on stomatal density agrees with the earlier reports of Sathiamoorthy (1987). The doubtful hybrids were subjected to flowcytometry analysis for confirmation of ploidy. The origin of pentaploids might be through a fusion of unreduced gametes from the tetraploid parent with reduced gametes from the diploid parent and the frequency of occurrence of unreduced gametes is genotype-dependent. Result of different ploidies of the selected hybrids as compared with nuclei isolated from diploid hybrid $(2 \mathrm{x})$ used as internal reference standard reveals that $\mathrm{H} \mathrm{504,} \mathrm{H} 540$ and $\mathrm{H} 573$ are clear pentaploids. Determination of nuclear DNA increased the reliability of ploidy and easy detection of mixiploids by Dolezel et al., (1997). Occurrence of pentaploids in $4 n \times 2 n$ cross was also earlier reported by many workers.

Hands of hybrids of phase I evaluation were bagged to study the female fertility/ parthenocarpiness. Among the hybrids evaluated in phase I generation, fifteen were found to be parthenocarpic and the rest viz., $\mathrm{H}$ 511, H 529, H 530, H 537, H 542, H 547, H 548, H 556 and H 571 were non parthenocarpic (Table 3 and 4). However, some of the parthenocarpic hybrids when pollinated artificially produced seed. Elain Apshara (2000) also observed similar results. Selection and utilization of parents with parthenocarpic pedigree might have contributed for enhanced parthenocarpy in the present investigation.

It also confirmed the role of dominant genes in controlling parthenocarpy by Simmonds (1953). Using flow cytomery, previous studies have shown inconsistencies in ploidy levels of banana accessions whose ploidy was determined based entirely on morphological traits by de Jesus et al., (2013), Dolezel et al., (1994), Irish et al., (2009), Nsabimana et al., (2006). Karamura et al., (2016) studies the ploidy level of 120 banana accession in the ex situ germplasm collection centre for the East and Central Africa through the flow cytometric analysis of the nuclear DNA content was used to determine the ploidy level of the accessions. Flow cytometry provides a rapid way of determining ploidy levels in this crop.

Out of 24 hybrids taken for Phase I evaluation, six diploids (AA and $\mathrm{AB}$ ), five triploids (AAA and $\mathrm{AAB})$, ten tetraploids (AABB) and three pentaploids (AAABB) were found. Among the 19 phase II hybrids evaluated, one diploid (AB), 
four triploids $(\mathrm{AAB})$ and fourteen tetraploids (AABB) were observed based on stomatal characters and morphological scoring and flowcytometry studies. Knowledge of the ploidy of bananas is valuable for banana breeding schemes as it involves interploidy crossed leading to several possible ploidy levels in the progeny. Flow cytometry provides a rapid way of determining ploidy levels in Banana.

\section{Acknowledgements}

The authors wish to thank and acknowledge the financial support of the Flemish office for Development cooperation and Technical Assistance (VVOB), Belgium and the International Network for the Improvement of Banana and Plantain (INIBAP) obtained through NRC for Banana.

\section{References}

Borges, F.1971. Study of female fertility in clones of plantains and cultivated bananas. Agronomica Trop. 21: 135-137.

Das, S. C. 2008. Breeding for resistance to nematodes and Fusarium Wilts in banana (Musa spp.) Ph.D. (Hort.) Thesis, Tamil Nadu Agricultural University, Coimbatore.

De Jesus, O N., de Oliveir, e Silva S, Amorim, E. P, Ferreira, C. F, de Campos, J.M.S., de Gaspari Silva, G, Figueira, F. 2013. Genetic diversity and population structure of Musa accessions in ex situ conservation. BMC Plant Biol. 14: 41.

Dolezel, J, 1998. Flow cytometry, its application and potential for plant breeding, Current Topics in Plant Cytogenetics Related to Plant Improvement, (LELLEY, T., Ed.), Universitätsverlag, Vienna (1998) 80-90.

Dolezel, J, Dolezelova, M, Novak, F. 1994. Nuclear DNA amount in diploid bananas(Musa accuminata and Musa balbisiana). Biol. Pplant. 36: 351-357.

Dolezel, J, Dolezelova, M, Roux N. and Houwe, I. V.1997.Use of flow cytometry for rapid ploidy determination in Musa species, Info Musa, 6:(1) 6-9.

Elain Apshara, S. 2000. Breeding bananas for resistance to nematodes and Sigatoka leaf spot. Ph.D. (Hort.) Thesis, Tamil Nadu Agricultural University, Coimbatore.

Irish, B.M, Crespo A, Goenaga, R, Niedz, R, Ayala-Silua, T. 2009. Ploidy level and grnomic composition of Musa spp. Accessions at the USDA-ARS. J. Agric Univ. Puerto Rico. 93: 1-21.

Karamura, D, Tumuhimbise, R, Muhangi, S, Nyine M, Pillay, M., Tendo R. S, Talengera, D, Namanya P, Kubiriba J, Karamura, E. 2016. Ploidy level of the banana (Musa spp.) accessions at the germplasm collection centre for the East and Central Africa. Afr. J. Biotechnol. 15(3): 1692-169.

Mustafa, Y. 2013. Plant responses at different ploidy levels, current progress in biological research, Marina-Silva-Opps (Eds.) ISBN: 978-953-51-1097-2. In Tech, DOI: $10.5772 / 55785$.

Nsabimana, A, Van Staden, J. 2006. Ploidy investigation of bananas (Musa spp.) from the National Banana Gerplasm Collection at Rubona-Rwanada by flow cytometry. S. Afr. J. Bot. 72: 320-305.

Pillay, M, Hartman J, Dimkpa C. and Makumbi D. 2003. Establishing the genome of Sukali Ndizi. Afr.Crop Sci. J, 11: 119124.

Pillay, M, Ogundiwin, E, Tenkuano, A and Dolezel J. 2006. Ploidy and gemome composition of Musa germplasm at the International Institute of Tropical Agriculture (IITA). Afr. J. Biotechnol. 5: 1224-1232.

Pillay, M, Tenkouano A. 2011.Genomes, Cytogentics and Flow Cytometry of Musa, in: Pillay, M, Tenkouano A (Eds.). Banana Breeding: Progress and Challenges, CRC Press, Boca Raton Fl.

Pillay, M, Tenkouano, A. and Hartman, J. 2002. Future challenges in Musa breeding. In: Crop Improvement: Challenges in the twenty- first century. Kang MS (Ed.), 
Food Products Press, Inc. New York. pp. 223-252.

Rowe, P. R.1984. Breeding bananas and plantains. Plant Breeding Rev, 2: 135155.

Sathiamoorthy, S. 1987.Studies on male breeding potential and certain aspects of breeding bananas. Ph.D. (Hort.) Thesis, Tamil Nadu Agriculture University, Coimbatore.

Sathiamoorthy, S.1973.Preliminary investigations on breeding potential of some banana clones. M.Sc. (Ag.) Thesis, Tamil Nadu Agriculture University, Coimbatore.

Shepherd, K. 1960. Seed fertility of edible bananas. J. Hort. Sci, 35: 6-20.

Shepherd, K. 1954. Seed fertility of the Gros Michel banana in Jamaica. J. Hort. Sci, 29: 1-11.

Simmonds, N. W. and Shepherd, K. 1987. A tentative key for identification and classification of Indian Bananas. National Research Centre for Banana (ICAR), Thiruchirapalli, India.

Simmonds, N. W. and Shepherd, K.1955. The taxonomy and origin of the cultivated bananas. J. Linn. Soci. Bot, 55: 302-12.

Simmonds, N. W.1948. The effects of ploidy upon the leaf of Musa. Ann. Bot, 12: 441453.

Simmonds, N. W.1953.Segregations in some diploid bananas. J. Genet, 51: 458-469.

Simmonds, N. W.1962.The evolution of the bananas. Longmans, Green \& Co, London.

Simmonds, N.W.1952.Experiments on the pollination of seeded diploid bananas. $J$. Genet, 51: 32-40.

Singh, H. P, Uma, S. and Sathiamoorthy, S. 2001. A tentative key for identification and classification of Indian Bananas. National Research Centre for Banana (ICAR), Thiruchirapalli, India. p 61.

Singh, H.P. and Uma, S.1996.Genetic diversity of banana in India. In: proceedings of the conference on "Challenges for banana production and utilization in 21 st century" held at Trichy, Sept. pp. 24-25.

Smith, M K, Hamill, S D, Langdon, P.W. and Pegg, K G.1993. Mutation breeding programme produces a plant with potential Fusarium wilt (Race 4) resistant Cavendish variety. Mutat. Breed. Newslett. 40: 4-5.

Suman, S, Rajak, K. K. and Kumar, H. 2012. Diversity of genome and ploidy in banana and their effect on tissue culture responses. Res. Environ. Life Sci. 5: 181183.

Tenkouano, A, Crouch, J H, Crouch, H.K, Vuylsteke, D. 1998. Ploidy determination in Musa germplasm using pollen and chloroplast characteristics. Hort.sci. 33:889-890.

Tenkouano, A, Pilly, M. and Ortiz, R.2011. Breeding techniques. In: Tenkouano A, Pilly (Eds.), Banana breeding progress and challenges. Taylor and Francis Group, pp. 181-202.

Van Duren, M, Morpurgo, R, Dolezel, J. and Afza, R.1996. Induction and verification of autotetraploids in diploid banana (Musa acuminata) by in vitro techniques. Euphytica. 88: 25-34.

Vandenhout, H, Ortiz, R, Vuylsteke, D, Swennen, R. and Bai, K.V.1995. Effect of ploidy on stomatal and other quantitative traits in plantain and banana hybrids. Euphytica. 83: 117-122.

\section{How to cite this article:}

Sukhen Chandra Das, T.N. Balamohan, K. Poornima and Van den Bergh, I. 2018. Studies of Ploidy Assessment in Some Synthetic Hybrids of Banana (Musa spp.). Int.J.Curr.Microbiol.App.Sci. 7(08): 3251-3264. doi: https://doi.org/10.20546/ijcmas.2018.708.347 